\title{
HISTORIA MODERNA EN TROCADERO, DE AUTORES Y TEMÁTICAS (1989-2008)
}

\author{
Jesús Manuel González Beltrán \\ Universidad de CÁdIZ
}

Durante estos primeros veinte años de vida de Trocadero, revista del Departamento de Historia Moderna, Contemporánea, de América y del Arte, se han publicado un total de 61 artículos que tienen como ámbito cronológico la Edad Moderna. La presencia de los modernistas ha sido continúa y bastante homogénea a lo largo de estas dos décadas, ya que tan sólo en los números 4 (monográfico sobre Historia de América), 5 (reservado a las actas del congreso sobre caciquismo y república) y 14-15 no consta aportación alguna. Mientras que el número 12-13, dedicado a la memoria del profesor del Departamento y catedrático de Historia Moderna José Luís Pereira Iglesias, recoge de forma exclusiva artículos modernistas, en total 18.

La revista ha sido, desde sus comienzos, un foro en el que los profesores del Departamento de Historia Moderna, Contemporánea, de América y del Arte de la Universidad de Cádiz, y otros autores ligados a dicho centro universitario, han expuesto los resultados de sus líneas de investigación. Tal es así que, como puede apreciarse en el gráfico 1, algo más de la mitad de los artículos, 33, el 54,1\% del total, han sido escritos por personas relacionadas, de una u otra forma, con el Departamento de Historia Moderna..., o con la Universidad de Cádiz. Los restantes 28 artículos se distribuyen entre autores que trabajan en otros centros de investigación españoles, en un 39,3\%, mientras que la presencia extranjera se queda en un mínimo 6,6\%.

Sobre este aspecto hay que destacar un cambio significativo que se ha producido en la evolución de la revista. Así, durante la primera década, los autores gaditanos presentaban un mayoría aplastante, con la autoría del 69\% de los artículos, mientras que en los últimos diez años son los autores no vinculados a la Universidad gaditana los 
que prevalecen con un 59,4\%, lo que implica una clara apertura de Trocadero y, algo más importante, el interés que despierta en compañeros de otros ámbitos territoriales el publicar en esta revista.

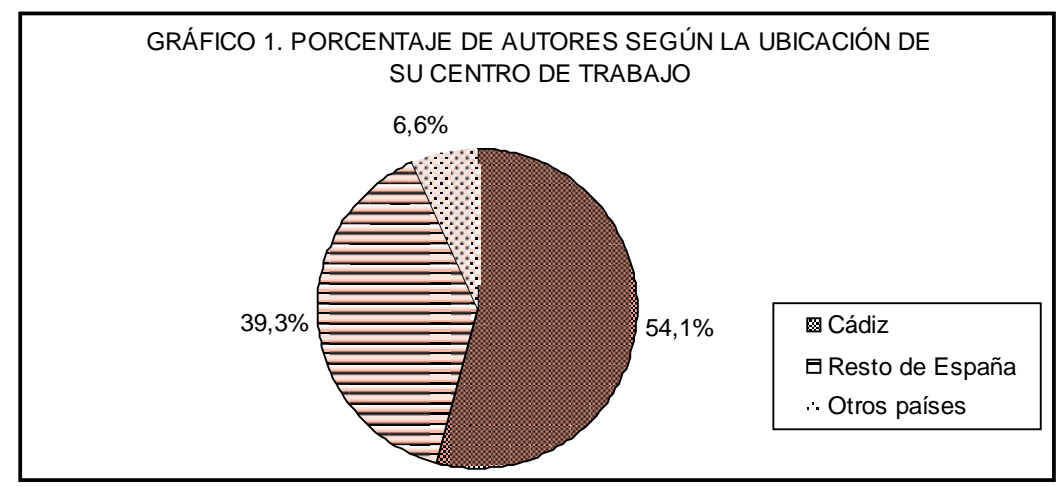

Muy en relación con la alta participación en la revista de autores relacionados con la Universidad de Cádiz es el hecho de que una parte significativa de los artículos de Historia Moderna publicados tengan como marco espacial de estudio la provincia gaditana, ya sea en su conjunto o tratando, de forma pormenorizada, alguna o algunas de sus localidades. Como se puede observar en el gráfico 2 hasta un 37,7\% de los artículos analizan cuestiones que ocurren o se manifiestan en el ámbito territorial gaditano. Y, aún se puede concretar más, ya que 14 de estas 23 investigaciones están dedicadas a la ciudad de Cádiz, cuestión por otra parte comprensible si se tiene en cuenta la importancia y significación de la mencionada localidad por su papel como puerto fundamental en el tráfico comercial con las Indias en el periodo de la Edad Moderna.

El resto de las provincias andaluzas ha tenido una escasa presencia en la revista, con apenas el 9,8\% de los artículos, copando el protagonismo la Historia Moderna de España, aunque quizás habría que matizar que se trata más bien de la Corona de Castilla. Son en total 28, un 45,9\% del total, las investigaciones que tienen un marco espacial español (sin contar Andalucía). Por último, se contabilizan 4 artículos, el 8,2\%, que se ocupan de territorios del extranjero, en concreto a la masonería en Portugal (Oliveira, 1, 1989), a las relaciones de Polonia con España (Zbigniew, 1, 1989), a la publicación en Inglaterra de un epicedio al Duque de Alburquerque (Charlo, 8-9, 1996-97) y al comercio de vinos de Inglaterra con Oporto y Jerez (Barros, 17, 2005).

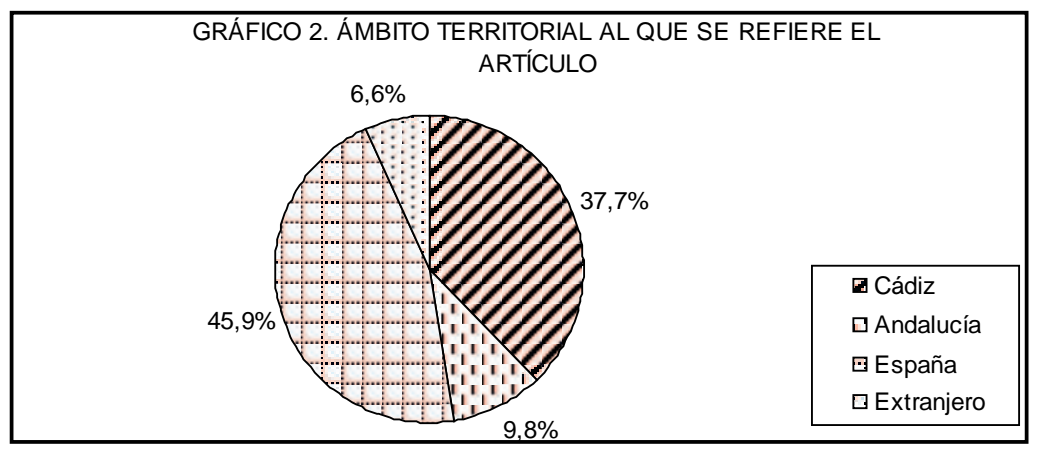


Al igual que ocurría con la procedencia de los autores, y en parte relacionado con ello, se advierte con respecto al marco geográfico de estudio una clara diferencia entre la primera y la segunda década de la revista Trocadero. Así, en los primeros diez años las investigaciones que conciernen a Cádiz alcanzaron el 44,8\% de todas las publicadas, mientras que en la segunda década el porcentaje ha quedado reducido al 31,2\%.

Un tercer aspecto que merece ser destacado en este análisis de los artículos de Historia Moderna aparecidos en las páginas de la revista Trocadero es el referido al marco cronológico, cuyos datos quedan plasmados en el gráfico 3.

Como puede apreciarse es el siglo XVIII el que ha recibido una mayor atención por parte de los autores, ya que son 26 las aportaciones, un 42,6\% del total, que se centran en dicha centuria. Porcentaje que se puede elevar hasta el $50 \%$, puesto que se podrían añadir casi todos los artículos que tratan los siglos XVII-XVIII, ya que 4 de los 6 que se ocupan de este periodo, aunque parten de la etapa final del XVII, para plantear la posible modificación de tendencias con el cambio de siglo, sus contenidos inciden especialmente en la centuria dieciochesca. Esta preponderancia del siglo XVIII se explicaría por dos cuestiones. La primera, el mayor peso que en la historiografía modernistas en general tiene la mencionada centuria, tanto por aspectos metodológicos (abundancia de fuentes, plantear los estudios como el resultado final de la evolución de toda la Edad Moderna), como por el atractivo de los temas (Ilustración, reformismo borbónico, inicio de los cambios estructurales) susceptibles de ser tratados. Y la segunda está más influenciada por el marco territorial. En efecto, siendo Trocadero una revista con una participación muy activa de autores vinculados a la Universidad de Cádiz y que, como se ha visto, hay una especial incidencia de investigaciones sobre la ciudad gaditana, es muy razonable ese predominio del siglo XVIII, pues es en esta centuria cuando la localidad de Cádiz alcanza su mayor esplendor y protagonismo al obtener la cabecera del monopolio comercial con las Indias. Tanto es así, que el 35\% de los artículos que tratan el siglo XVIII tienen como ámbito espacial a la ciudad de Cádiz.

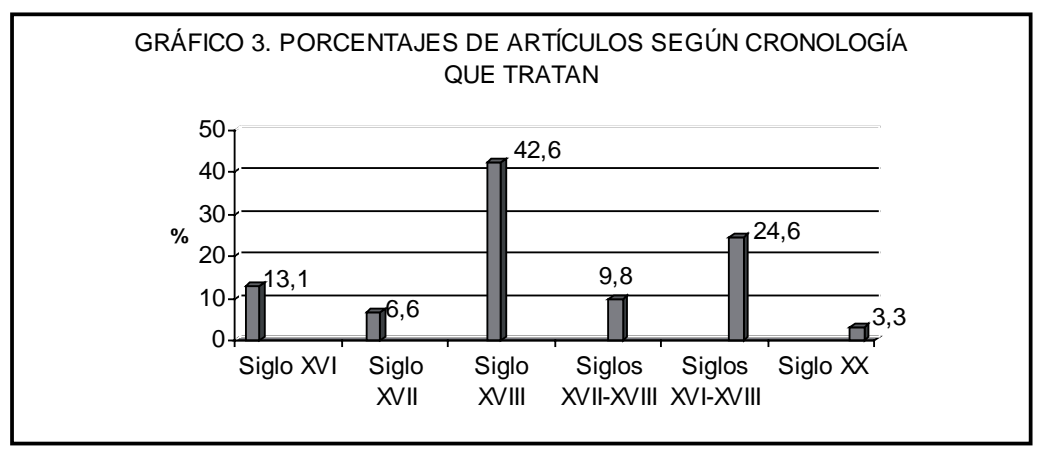

En comparación con el siglo XVIII las otras dos centurias de la Edad Moderna apenas reciben un tratamiento minoritario. Se contabilizan 8 artículos referidos al siglo XVI, un 13,1\% del total, y tan sólo 4 investigaciones, un 6,6\%, que traten el siglo XVII. Hay dos artículos que se enmarcan en el siglo XX, ya que analizan el oficio de historiador (Rodríguez Cancho, 12-13, 2000-01) y los posicionamientos de los histo- 
riadores de la ciencia (Villas, 12-13, 2000-01). Por último, el segundo bloque temporal en importancia es aquel que tiene como marco cronológico a los tres siglos de la modernidad en su conjunto, a la Edad Moderna en su totalidad. Son 15 aportaciones que se corresponden con el 24,6\% de todas las publicadas.

En este apartado dedicado a la cronología hay que reseñar que no existen diferencias significativas entre la primera y la segunda década de la revista, estado todos los periodos mencionados equitativamente representados en ambas, a lo sumo se podría resaltar el siglo XVI, que pasa de 2 a 6 artículos.

El último aspecto de análisis es quizás el más interesante, ya que hace referencia a la temática de los artículos publicados en Trocadero. En base a la simplificación expositiva se han dividido los asuntos y temas tratados en 7 grupos: Historia Social, Historia Económica, Historia Cultural, Religión, Muerte, Demografía y un último de otros varios. Los datos de la distribución porcentual pueden comprobarse en el gráfico 4.

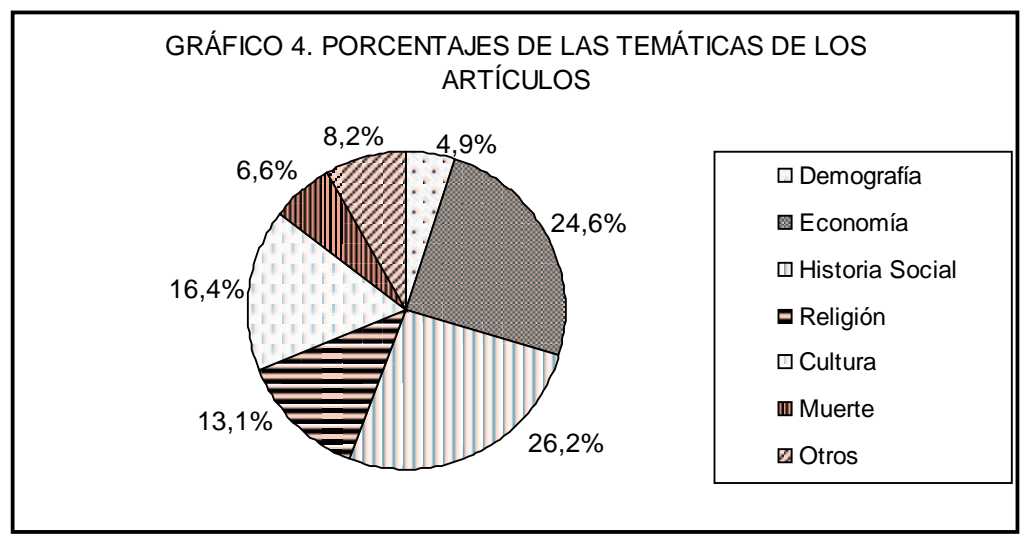

Es la temática de Historia Social, con 16 artículos, un 26,2\% del total, la que ostenta una mayor presencia en la revista. Se trata de una Historia Social renovada, aunque se sustente sobre la base deslizante de una controvertida falta de definición motivada por su múltiple funcionalidad, y, a la vez, con unos objetivos bien definidos: plantear interpretaciones tanto sobre los mecanismos de pervivencia y reproducción del sistema social como sobre los factores de cambio o alteración del mismo. No hay ninguna aportación de este campo que se acerque al conjunto de la sociedad, todas hacen referencia a algún grupo específico. Así, se contabilizan 5 artículos que se ocupan del clero, destacando especialmente los trabajos de Barrio Gozalo sobre los aspectos socioeconómicos de los obispos gaditanos durante la Edad Moderna (12-13, 2000-01) y el de Álvarez Santaló dedicado a la situación económica del clero secular de Sevilla en el Antiguo Régimen, utilizando para ello 142 inventarios del periodo 1700-1834 (8-9, 1996-97). Otras 5 investigaciones tienen por objeto a la nobleza, ya sea un linaje concreto, como el de Toledo-Golfín analizado por Álvarez de Toledo (12-13, 2000-01 y 17, 2005) o el de Ayllón-Mayorgas examinado por Piqueras y Morgado (18, 2006); una orden nobiliaria, como la de Damas de la reina María Luisa, estudiada por Molas Ribalta (12-13, 2000-01); o la compra de empleos militares para “colocar” a miembros 
segundones de la nobleza, aspecto visto por Sanz de la Higuera (20, 2008). Si los estamentos privilegiados contabilizan 10 artículos, el estamento general o tercer estado recibe una menor atención, tan sólo 6 , a pesar de los numerosos grupos que componen el mismo y las muchas posibilidades que depara su estudio. La heterogeneidad de estas aportaciones es total, fiel reflejo del propio estamento. El análisis de las redes de poder de la elite administrativa, financiera y comercial Navarra en el siglo XVIII, con una excelente introducción metodológica, es tratado por Imízcoz Beunza (19, 2007); mientras que Fernández Pérez (6-7, 1994-95) ofrece un estudio prosopográfico de los comerciantes gaditanos del siglo XVIII, incidiendo en la estructura familiar de dicho grupo socioprofesional. Resulta raro pero ésta es la única aportación que trata desde un punto de vista social a los comerciantes de Cádiz, máxime cuando es elemental el protagonismo de los mismos en la sociedad gaditana de la Edad Moderna. Descendiendo en la escala social, los grupos populares han recibido estudios de Morgado (12-13, 2000-01), dedicado a la pobreza y los grupos pauperizados en Cádiz; González Beltrán $(17,2005)$, que analiza las condiciones de trabajo y la conflictividad laboral, a finales del siglo XVIII, de los trabajadores del campo; y Amelang (16, 2004), que plantea cuestiones metodológicas sobre la autobiografía popular. Para terminar, los extranjeros son examinados en función de cómo les afectó la legislación emitida en la década final del siglo XVIII a fin de controlarlos, en especial a la colonia francesa, artículo propio de González Beltrán (8-9, 1996-97).

Las aportaciones de Historia Económica son también considerables, alcanzando el número de 15, un 24,6\% del total. La impronta de la ciudad de Cádiz, con su protagonismo en el monopolio, durante el siglo XVIII, del tráfico mercantil con las Indias, queda plasmada en una actividad económica recurrente: el comercio. En efecto, hasta 9 de los 15 artículos mencionados tienen por objeto estudiar distintos aspectos comerciales, ocupándose 7 de ellos del comercio gaditano o el que tiene lugar con la América española. Con respecto a éste último habría que destacar la investigación de García-Baquero (12-13, 2000-01) sobre los debates y la postura personal del consejero de Indias D. Fernando Magallón en relación al decreto de Libre Comercio de 1778; y el análisis llevado a cabo por Bustos (8-9, 1996-97) de las opiniones de los tratadistas de la Edad Moderna sobre los males de España en relación con el comercio con las Indias y la participación en el mismo de los extranjeros. En cuanto a la ciudad de Cádiz y su actividad mercantil señalar los trabajos de Carrasco (8-9, 1996-97) sobre la correduría de lonja y los corredores, en comparación con la localidad de Jerez de la Frontera; y de Morgado (10-11, 1998-99), escrutando las relaciones comerciales entre Cádiz y el norte de África durante el siglo XVII. Novedoso resulta el estudio de Herrero Gil (20, 2008) relativo a la implantación del correo marítimo España-Indias durante el reinado de Carlos III, con sede administrativa en Cádiz. En comparación con el comercio las otras actividades económicas apenas tienen relevancia. Ningún artículo se ocupa de la agricultura y sólo uno del sector industrial, cuyo autor, Torrejón (3, 1991), informa de las fábricas existentes a finales del siglo XVIII en la real Isla de León, importante centro logístico de la Armada. Las aportaciones restantes de temática económica tienen relación con cuestiones financieras o fiscales. La estructura del capital en Jerez de la Frontera a través del análisis de 355 inventarios post mortem del periodo 1750-1790 es ofrecida por González Beltrán (12-13, 2000-01). Pereira Iglesias (6-7, 1994-95 y 
8-9, 1996-97) ilustra sobre los censos consignativos con garantía real, desde los posicionamientos del pensamiento económico y a través del examen de sus características y formalidades. Y, finalmente, Cortes Peña (12-13, 2000-01) estudia la percepción del diezmo en la comarca almeriense de los Vélez, fruto de conflictos entre los señores jurisdiccionales y las autoridades eclesiásticas.

El tercer campo temático en número de artículos, con 10, el 16,4\% del total, corresponde a la Historia Cultural. Una temática amplia y en la que destaca la heterogeneidad de las aportaciones. Tanto es así que junto a estudios clásico de esta materia como puede ser el de La Pascua Sánchez $(1,1989)$ relativo a los niveles de alfabetización, cuantificados en base a la firma de los testamentos, en localidades de la provincia de Cádiz, encontramos nuevas vías de investigación como las planteadas por Pérez Samper (12-13, 2000-01), sobre las pautas de comportamiento social a través del análisis del libro de Yelgo Bázquez Estilo de servir a Príncipes, publicado en 1614; o por Morgado (20, 2008), que examina la pervivencia de los mitos, del imaginario sobre monstruos marinos a lo largo de la Edad Moderna. El análisis del discurso, de los textos, también ha sido tratado en la revista Trocadero, en cuya línea merecen reseñarse las aportaciones de Dahlman (10-11, 1998-99) sobre el discurso pedagógico, en el siglo XVIII, del fraile benedictino Martín Sarmiento, en especial a través de su obra Tratado sobre la educación; y Alfonso Mola y Martínez Shaw (12-13, 2000-01) que señalan el carácter reformador de Felipe $\mathrm{V}$ tal como lo muestran dos discursos laudatorios presentados en 1779 a un concurso de elocuencia organizado por la Real Academia de la Historia.

Aunque podrían haberse incluido en el apartado anterior dedicado a la Historia Cultural, los 8 artículos con temática religiosa, un 13,1\% del total, se presentan, al objeto de recalcar su incidencia en la revista, conformando un bloque separado. La mitad de los estudios se deben a un mismo autor, Morgado, que trata la fundación de oratorios (1, 1989); la actividad benéfica (3, 1991); las demandas de divorcio (6-7, 1994-95); y los manuales de confesores (8-9, 1996-97). Un lucido artículo es el presentado por Álvarez Santaló $(1,1989)$ relativo a la influencia en la organización y modelación de la sociedad barroca de los denominados libros de devoción, uno de los cuales, Luz de los Vivos de Palafox, procede a analizar. Y no menos interesante resulta la investigación de Martínez Millán (6-7, 1994-95) en la que se analiza el confesionalismo de Felipe II y la incidencia que en la estructuración e implantación del mismo jugó la Inquisición.

Hay 4 aportaciones, un 6,6\% del total, que tratan cuestiones relativas a la muerte (práctica testamentaria, enfermedad, método de enterramiento,...), las cuales también podrían haber sido incluidas en la Historia Cultural o en la ya en declive y criticada Historia de las Mentalidades. De estos artículos recalcar el de La Pascua Sánchez (8-9, 1996-97) que analiza el discurso sobre la muerte desde la triple perspectiva de amenaza futura, realidad presente y pasada memoria colectiva; y el de Álvarez Santaló (12-13, 2000-01) sobre la percepción de la enfermedad, el dolor y la muerte en el siglo XVII, siguiendo el contenido de la biografía de la beata de Aracena, escrita por fray Antonio de Lorea.

Una materia que en su momento fue fundamental y que hoy en día prácticamente ha quedado en un plano totalmente secundario es la Demografía Histórica, de la cual se contabilizan 3 artículos en estos 20 años de la revista Trocadero. El más interesante, 
por la amplitud de los datos ofrecidos y por servir de base para investigaciones posteriores, es el realizado por Bustos Rodríguez y otros $(2,1990)$ sobre la evolución de la población de la provincia de Cádiz durante el conjunto de la Edad Moderna, para lo cual utiliza los datos de los censos disponibles y de las series parroquiales completas de 8 localidades.

Para finalizar con el análisis de las temáticas de los artículos aparecidos en Trocadero se ha conformado un bloque de varios, en el que, junto a una aportación metodológica sobre la función del historiador, encontramos dos dedicadas a los Estudios de Género y otras dos a la Historia Militar.

En este aspecto de los temas tratados tan sólo en dos casos se puede señalar una cierta diferencia entre la primera y la segunda década de la revista Trocadero. El primero lo constituye la Historia Social, aumentando considerablemente el número de aportaciones en la segunda década, al pasar de 4 a 12 artículos. Contrariamente, los estudios sobre cuestiones religiosas han perdido peso y, así, los 7 artículos de los primeros diez años han quedado reducidos a tan sólo 1 en la segunda década. 
